\title{
The Research of CPS System Driven by Blockchain
}

\author{
Feng Zhu ${ }^{1, a)}$, Jingwei Qiao ${ }^{2, b)}$ \\ ${ }^{1}$ School of Computer Science, Guangdong University of Technology, China. \\ ${ }^{2}$ School of Information Engineering, Guangdong University of Technology, Guangzhou 510006, China \\ a) Feng Zhu: 302761359@qq.com \\ b) Jingwei Qiao: 2221393385@qq.com
}

\begin{abstract}
The CPS system senses and recognizes the physical world through numerous node devices and processes the collected data accordingly, then it can achieve the interconnection of the physical world and the virtual network world. However, under the existing conditions, the CPS system has not yet realized the interconnection between nodes and nodes and the physical world. Block chain technology is a distributed ledger in which nodes can freely enter and exit. This article proposes to combine the block chain with the CPS system to improve the efficiency and security of the CPS system. Through theoretical analysis and experiments, it is proved that the combination of block chain and CPS system can effectively improve the scalability of CPS system and improve the ability of CPS system to communicate with the physical world. Through the combination of the public chain and private chain of the block chain, the production speed of the block is improved, and the actual demand of the CPS system is better satisfied.
\end{abstract}

Key words: CPS system; interconnection; data accordingly; Block chain technology.

\section{INTRODUCTION}

Cyber-Physical Systems (CPS) is a highly integrated multidimensional computer unit and physical object in a networked environment. It consists of a node layer, a network layer, a resource layer, and a service layer and its core foundation are the node layer. The system implements sensing, identification and controls functions at the node level. In the "White Paper on Information Physics Systems", the CPS system structure is defined as three layers: unit level, system level, and system level of the system. The unit level is the basic unit of the CPS system and remains the core layer of the system.

The CPS system is a centralized structure. The data exists in a trusted third-party server. At the same time, the CPS system is also a closed system. It lacks connection with the external world. The data in the external world is generally carried by $U$ disk and other mobile devices. Transmission, however, must be infected with these transmission media, then all related devices in the cps system will be compromised, and then make the entire cps system has been incalculable loss.

Block chain is the underlying technology of Bitcoin. It is a distributed ledger. Data is recorded by all participating nodes in the block chain system. Each node owns all the data in the system, and only the majority of nodes acknowledge it. Only when the data is authentic can it be recorded in the system. Otherwise, the data is rejected and the corresponding node providing the data is punished. A block chain is a data structure in which blocks are sequentially linked together in chronological order. A block refers to a collection of data and is a basic unit for forming a block chain. Each block consists of two parts, which are the block header and the block body. The block header can provide information linked to the previous block and provide integrity for the block chain; the block body records updated data information in the network. The blockchain structure is shown in Figure 1. 


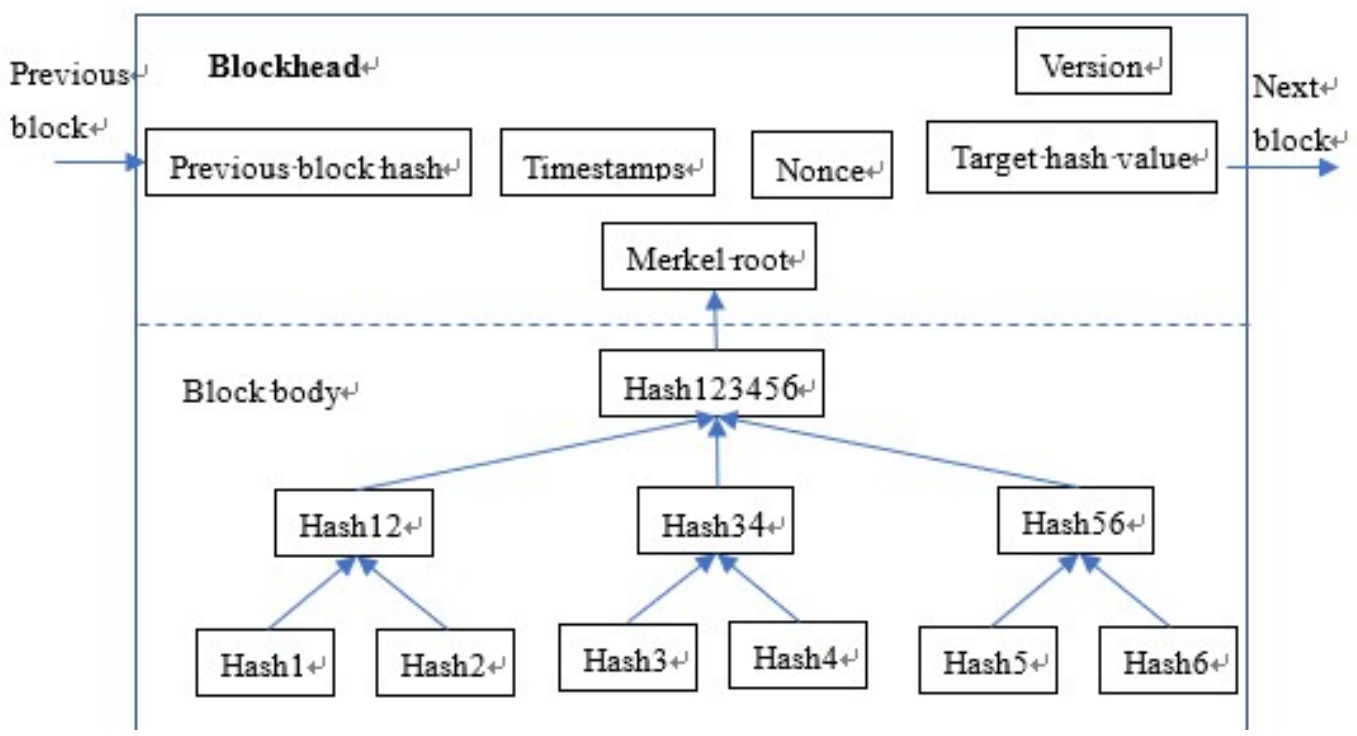

FIGURE 1. Structure of block

At present, there are few researches on CPS in blockchain, but there are still many researches on blockchain in the field of IoT. The following is a comparison between the similarities and differences between the Internet of Things and CPS systems.

TABLE 1. IOT\&CPS

LOT CPS

Difference achieving awareness, control of the achieving awareness, control of the
composition of the few, that is, the need to deal with less data
The need to implement information transfer, in addition, the need for coordination capabilities, greater demand for computing power, so as to achieve autonomy

\begin{tabular}{|c|c|}
\hline \multirow{3}{*}{$\begin{array}{l}\text { Same } \\
\text { point }\end{array}$} & The network relates to the nodes of the system to form a whole \\
\hline & More nodes \\
\hline & Node security protection \\
\hline
\end{tabular}

\section{RELATED WORK}

\section{Internet of Things-Related Research}

At present, the research of blockchain technology in manufacturing, communications and other fields is still in its infancy, and there is no practical verification of its theoretical feasibility. However, there are many studies in the financial field, such as cross-border payment, bank credit, and so on. This article studies the usability of blockchain technology in CPS systems. However, because there are few studies in this area, the article first studied the research status of blockchain technology in the field of IoT.

The literature [1] proposed a method using credibility scores to ensure the orderly operation of blockchain networks. In this method, the greater the number of contracts signed by a node, the higher the credibility score, and the assignment of node rights according to the node's credibility score. The literature [2] pointed out that the method of proof of workload will cause a Centralization to a certain extent. Therefore, the article proposes a mechanism for binding physical entities to virtual identities, which can be implemented while maintaining anonymity. 
Responsibility, this method ensures the security of the node, and at the same time it can achieve a more fair and democratic approach to the operation of the blockchain system.

The literature [3] regards the blockchain as an innovation to manage the digital society. In the context of the energy Internet, it designs a hybrid blockchain storage model based on the blockchain technology in terms of its security and privacy issues. In order to improve the overall efficiency of the Internet operation, realize decentralized supervision and promote the credible, safe and efficient operation of the massive data storage of the energy Internet

The literature [4] directly uses Ethereum's smart contract to write its own code to implement the configuration of the Internet of Things devices and the synchronization between devices to prevent denial of service attacks.

In summary, blockchains have proposed various improved methods in the research of the Internet of Things, so that the blockchain can be better applied. Based on the research of blockchain in the field of Internet of Things, this paper proposes a blockchain solution to the problems in CPS.

\section{Blockchain Related Research}

Blockchain is an important concept of Bitcoin. It is Bitcoin's underlying technology. Its essence is a decentralized database. Everyone can participate in the blockchain network. Every device can act as a blockchain. A node in the network, each node is allowed to obtain a complete copy of the database, based on a set of consensus mechanisms between the nodes, through the competition to maintain the entire blockchain. The distributed structure of the blockchain allows the storage of data to be distributed on various nodes of the network instead of being centrally recorded on the centralized server host. The distributed architecture increases the robustness of the entire blockchain network system and does not affect the operation of the entire network system due to the failure of some nodes. Blockchain system has anti-attack, data confidentiality, self-healing toughness [5], these features can prevent data from being maliciously tampered with, protect the user's privacy information and can improve the system's selfhealing capabilities.

\section{BLOCKCHAIN COMBINED WITH CPS}

In response to the layering in the CPS system mentioned in the first part, we propose to combine the layered features of the CPS system with the blockchain technology to solve the problems in the CPS.

The CPS system is divided into three layers: the unit level, the system level, and the system level of the system. The position of each layer in the system and its importance to the system are different. The hierarchical structure of CPS is shown in Figure 2.

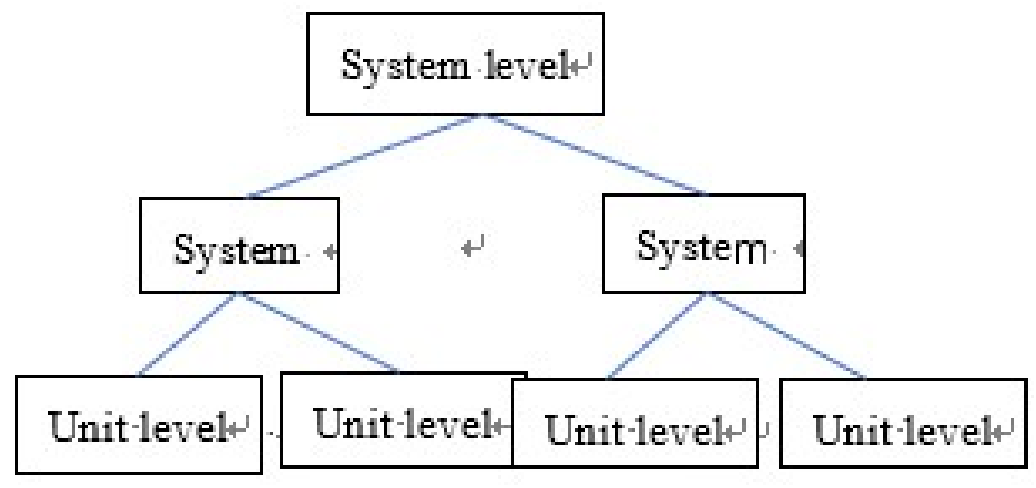

FIGURE 2. Structure of CPS

From the above figure, we can see that each level of the system is complementary. This paper proposes to implement hierarchical blockchain technology management according to the hierarchical structure of CPS. The specific description is as follows:

The unit level is the smallest and non-divisible unit of the CPS system. It is the core unit that the CPS system perceives the physical world and interacts with the physical world. When these unit-level CPSs that undertake a 
single task are attacked or destroyed by humans, they cannot pose a threat to the security of the entire CPS system. Therefore, the impact of our definition of cell-level CPS on the entire CPS system is relatively small.

System-level CPS is composed of multiple cell-level CPSs. It can realize automatic flow of a large range of data through industrial networks. It can accomplish certain functions and realize the scheduling and configuration of production factors in an industrial production environment. System-level CPS is more important for the entire CPS system. Once the system-level is threatened by security, it will threaten the entire industrial production process

The System of System (SOS) of the system is an organic combination of multiple system-level CPSs. It is a cross-system, cross-platform interconnect, interoperability, and interoperability. It is a global scope of execution, scheduling, and configuration. The security of this layer is the entire CPS system is a top priority.

According to the above analysis of the CPS system, we propose that different levels of nodes in the CPS system be given different trusted weights in the blockchain system. At the same time, in the layered CPS system, private blockchains and public blocks are used. Chains are used together. In the process of data consensus between nodes in a blockchain system, the nodes in the cell-level CPS give a lower weight, the system-level CPS gives a higher weight, and the system-level CPS nodes give the highest weight. When these nodes in the CPS system interact with each other, they analyze their influence on the system according to the trusted proportion, and then make a final evaluation of the data. If they are trusted, they are recorded in the blockchain system. If it is not trustworthy, then give up the record of the data, and make a corresponding reduction in the credibility of the corresponding node. During the process of data recording in the system, the system selects nodes according to the node's credibility score. The higher the node's credibility score, the greater the probability that the node is selected as a billing node.

\section{EXPERIMENTS AND ANALYSIS}

The work steps of the blockchain combined with the CPS system are as follows:

The information exchange between the unit-level CPS nodes generates interactive data.

The data generated at the cell level is encrypted and transmitted to the system-level CPS control system, namely the public blockchain.

According to the level of the two-level node in the system and the credibility of the node, the node is calculated by weighted credibility, and the node that records the data is selected from the higher-graded nodes.

The selected node records the system information data, which is agreed by the entire network node, encrypted and stored in the block after confirmation by the entire network node; and the credibility score of the correct data node is recorded. Update, increase the credibility of the node.

If the node makes false information records or maliciously generates redundant information, it passes the system consensus process. The node's credibility score will be reduced, reducing the node's credibility in the system.

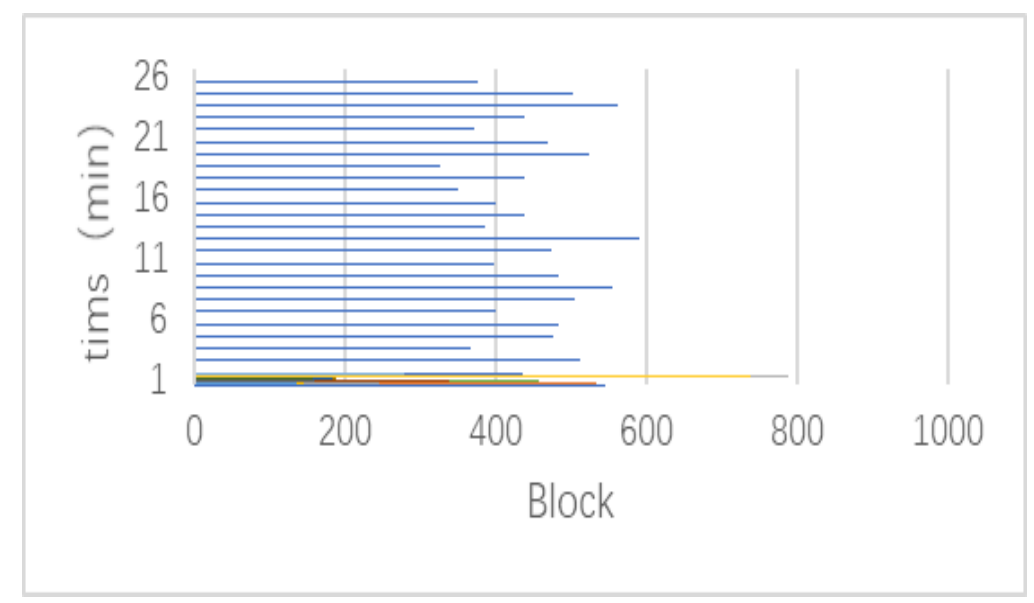

FIGURE 3. Performance Analysis Chart

As can be seen from the above figure, the private chain operates with the public chain, and the block generation is maintained at about 10 seconds. Compared with the Ethereum project, the efficiency has been greatly improved. 


\section{CONCLUSION}

Through the research of the article, it is found that under the conditions of the combination of the public chain and the private chain, the speed of the block generation in the blockchain is improved, and the work efficiency of the system is improved. In addition, the nodes in the CPS system under the control of the blockchain can freely enter and exit the system, which effectively improves the scalability of the system, breaks the closedness of the system, and truly realizes the intelligent manufacturing industry. Through the discussion on the application of blockchains in the Internet of Things and CPS systems, it can be seen that the development of blockchains in the manufacturing industry is very promising. In the future, it can promote the manufacturing industry to become truly fully automatic and intelligent.

\section{REFERENCES}

1. Dongqi Fu and Liri Fang, "Blockchain-based trusted computing in social network," 2016 2nd IEEE International Conference on Computer and Communications (ICCC), Chengdu, 2016, pp. 19-22.

2. M.Borge, E. Kokoris-Kogias, P. Jovanovic, L. Gasser, N. Gailly and B. Ford, "Proof-of-Personhood: Redemocratizing Permissionless Cryptocurrencies," 2017 IEEE European Symposium on Security and Privacy Workshops (EuroS\&PW), Paris, 2017, pp. 23-26.

3. L. Wu, K. Meng, S. Xu, S. Li, M. Ding and Y. Suo, "Democratic Centralism: A Hybrid Blockchain Architecture and Its Applications in Energy Internet," 2017 IEEE International Conference on Energy Internet (ICEI), Beijing, 2017, pp. 176-181.

4. S. Huh, S. Cho and S. Kim, "Managing IoT devices using blockchain platform," 2017 19th International Conference on Advanced Communication Technology (ICACT), 2017, pp. 464-467.

5. Blockchain Technology in Energy Internet: Initial Exploration of Research Framework and Typical Applications Zhang Ning 1, Wang Yi 1, Kang Chongqing 1, Cheng Jiangnan 1, He Dazhao 2 